\title{
Decision-making in a synthetic cell: the limits of biological computation
}

\author{
Ferdinand Greiss ${ }^{1}$, Shirley S. Daube ${ }^{1}$, Vincent Noireaux ${ }^{2}$, Roy Bar-Ziv ${ }^{1, *}$ \\ 1Department of Chemical and Biological Physics, Weizmann Institute of Science, \\ Rehovot 76100, Israel \\ ${ }^{2}$ Department of Physics, University of Minnesota, Minneapolis, Minnesota 55455, \\ USA \\ ${ }^{*}$ Corresponding author
}

\begin{abstract}
We measured the dynamics of decision-making by a minimal bistable gene network integrated in a synthetic cell model, free of external perturbations. Reducing the number of gene copies from $10^{5}$ to about 10 per cell revealed a transition from deterministic and slow computation to a fuzzy and rapid regime dominated by singleprotein fluctuations. Fuzzy computation appeared at DNA and protein concentrations 100-fold lower than necessary in equilibrium, suggesting rate enhancement by coexpressional localization. Whereas the high-copy regime was characterized by a sharp transition, hysteresis and robust memory, the low-copy limit showed incipient strong fluctuations, switching between states, and a signature of cellular individuality across the decision-making point. Our work establishes synthetic cells operating rapidly at the single molecule level to integrate gene regulatory networks with metabolic pathways for sustained survival with low energetic cost.
\end{abstract}

\section{One Sentence Summary}

Decision-making in a synthetic cell can be slow and precise or rapid and probabilistic by reducing the number of computing molecules by five decades down to singlemolecule fluctuations. 


\section{Main Text}

Making well-informed decisions takes time and energy. The fundamental connection between those three aspects is exemplified by recognition memory in cognitive tasks (1), optimality in molecular recognition as an error-correction mechanism $(2,3)$, and cellular adaptation in chemo-sensing (4). Decisions on a cellular level are made by regulatory proteins that integrate information from the environment and elicit a response by modulating RNA and protein production. In each cell, the copy number of regulatory molecules could vary between one to a few hundred, subjecting the information processing to random fluctuations $(5,6)$. The fluctuations can stem from production and degradation of proteins, and the binding and unbinding of proteins to and from their regulatory sites on DNA. Theoretical considerations suggest that a decision becomes exponentially more stable as the copy number of regulatory elements, hence metabolic load, increases $(7,8)$.

Decision-making of genetic regulatory networks (GRN) should be precise and deterministic when the process is averaged over many computing molecules. However, in the small-number limit, fluctuations in gene-expression with a few computing molecules, hence little averaging, may reduce the precision and lead to fuzzy computation (Fig. 1A-B). By measuring the characteristics of a GRN in the high and low copy number regime, we addressed the following questions: Can we measure the transition from deterministic to fuzzy decision-making dynamics driven by a GRN? Can we recognize a fundamental tradeoff between the two regimes? And how do single-molecule fluctuations influence the decision-making?

In order to address these questions experimentally, a programmable cell-free system of gene-expression reactions with constant protein turnover is required (9). To that end, we built synthetic cell models devoid of extrinsic noise stemming from DNA replication, genetic cross-talk, or cell size variation (10-12). Each cell had a DNA compartment with a $20 \mu \mathrm{m}$ radius and $3.5 \mu \mathrm{m}$ height, connected by a thin capillary ( $\mathrm{L}=90 \mu \mathrm{m}$ and $\mathrm{W}=7 \mu \mathrm{m})$ to a reservoir of cell-free extract to support transcriptiontranslation (TXTL) reactions (Fig. 1C, Fig. S1) (13). The compartment volume was $3.810^{3} \mu^{3}$, roughly 1000 -fold larger than a typical bacterial cell. The capillary allowed free diffusion in and out of the compartment, creating TXTL dynamics with 
an apparent protein lifetime of $\tau=\pi R^{2} L / W D=8 \mathrm{~min}(13)$, with $\mathrm{D}=30 \mu \mathrm{m}^{2} / \mathrm{s}$ as the typical protein diffusion coefficient. We used an elastomer to create the compartments, bonded to a coverslip to detect the synthesis of fluorescent reporter proteins from the DNA attached on the glass substrate (Methods).

We based our genetic model system on a bistable GRN built from the core elements of the lambda bacteriophage regulatory network (Fig. 1B). In its native environment, this GRN controls the phage's decision to lyse the cell or integrate its genome into the bacterial host genome thus entering the lysogenic state. This state can be maintained for more than $10^{5}$ generations by the presence of only a few hundred copies of proteins before switching back to the lytic phase $(10,14-17)$. The minimal GRN consists of two transcription factors, $\mathrm{Cl}$ and Cro repressors, which mutually inhibit each other's production by binding to their respective promoters $\left(P_{R}\right.$ and $\left.P_{R M}\right)$ (Fig. 1B) (16). By analogy to digital electronics, an ideal bistable GRN can be viewed as a latch circuit that activates either of two promoters and remembers the active promoter until toggled (Fig. 1B). $\mathrm{Cl}$ has been shown to be the main regulator, responsible for entering and maintaining the lysogenic state, while Cro buildup serves a tipping point to decisively and irreversibly enter the active $P_{R}$ promoter (lytic) state (18). This inherent asymmetry between $\mathrm{Cl}$ and Cro is due to the promoters' architecture including auto-inhibition and auto-activation loops (16). To toggle the promoter activities, we used a temperature-sensitive $\mathrm{Cl}\left(\mathrm{Cl}^{\mathrm{ts}}\right)$ mutant that tunes its deactivation rate with a rise in temperature from $30^{\circ} \mathrm{C}$ (no deactivation) to $41^{\circ} \mathrm{C}$ (fast deactivation) $(19,20)$.

We immobilized the DNA constructs with the bistable GRN on the surface of the compartments. At the high-density regime, we packed roughly $10^{5}-10^{6}$ copies (21) in a DNA brush patterned on a circle of $14 \mu \mathrm{m}$ diameter (Methods). We placed the gene coding for green fluorescent protein (GFP) in tandem with the cro gene, as a reporter of the activity of the strong $P_{R}$ promoter $(16,20)$ (Fig. 1D), and recorded the signal using epi-fluorescence microscopy. We observed a smooth and noiseless GFP signal, peaking after $\sim 2$ hours and slowly reaching steady-state values after two more hours (Fig. 1D). 
For the low-copy regime, we immobilized an average of $\sim 20$ copies of the bistable GRN in each compartment, which amounted to a very low effective DNA concentration of $\sim 10 \mathrm{pM}$ (Fig. 1E and Fig. S2). To allow the detection of single proteins, we fused the $\mathrm{cl}^{\text {ts }}$ gene under the weak $\mathrm{P}_{\mathrm{RM}}$ promoter $(16,20)$ to the fastmaturing fluorescent protein mVenus (22), and circularized the DNA constructs to minimize degradation by exonucleases in the E. coli extract (Methods, Fig. S3, Table S1). We employed total internal reflection fluorescence (TIRF) microscopy with a penetration depth comparable to the typical dimension of DNA molecules to observe localization of the proteins close to the coverslip surface.

After introducing the cell-free extract, we could observe the appearance of discrete $\mathrm{Cl}^{\mathrm{ts}}$-mVenus fluorescent spots that were single-peaked and rapidly bleached with a lifetime of $\sim 4 \mathrm{sec}$ (Fig. S4). The fast bleaching removed all newly produced proteins that localized to the surface. We could not reliably detect repeated binding of proteins to specific locations due to nonspecific adsorption on the surface upon heatinactivation of $\mathrm{Cl}^{\text {ts }}$-mVenus (Fig. S5). Therefore, we integrated the number of produced proteins inside the compartment independently of the location into $15 \mathrm{sec}$ time intervals, reporting on the overall $\mathrm{P}_{\mathrm{RM}}$ activity of the minimal cell model (Fig. 1E) $(20,23,24)$. In sharp contrast to the high-density regime, we observed strong fluctuations of protein production rates that reached a first maximum after $20 \mathrm{~min}$.

Because we optimized the cell extract such that the basal protein production rate varied by no more than $\sim 10 \%$ for $31-41^{\circ} \mathrm{C}$ (Fig. S6), any change in signal properties as a function of temperature could be attributed to a response of the GRN to a change in $\mathrm{Cl}^{\text {ts }}$ deactivation rate. Based on the GRN architecture studied in bacteria $(10,16,20)$, we anticipated initial low $P_{R M}$ and low $P_{R}$ activities at low temperature, where stable $\mathrm{Cl}^{\text {ts }}$ first represses $\mathrm{P}_{\mathrm{R}}$ and then self-represses $\mathrm{P}_{\mathrm{RM}}$ at the mutual promoter sites (Fig. 2A, state 1). An increase in $\mathrm{Cl}^{\text {ts }}$ deactivation rate at higher temperature should reduce the occupancy of $\mathrm{Cl}^{\text {ts }}$ at the promoters, leading to selfactivation of $\mathrm{Cl}^{\text {ts }}$ and gradual production of Cro from $\mathrm{P}_{\mathrm{R}}$ (Fig. 2A, state 2). At higher temperatures, complete deactivation of $\mathrm{Cl}^{\text {ts }}$ leads to fully active $\mathrm{P}_{\mathrm{R}}$ (Fig. 2A, state 3). 
At the high-density regime with GFP, hence Cro, reporting on $\mathrm{P}_{\mathrm{R}}$ activity, no signal was recorded at $31^{\circ} \mathrm{C}$, indicating an active $\mathrm{P}_{\mathrm{RM}}$ promoter (Fig. 2B, upper panel, Fig. S7, and Fig. 2A, state 1). We observed a decisive transition between $38^{\circ} \mathrm{C}$ and $39{ }^{\circ} \mathrm{C}$ to high GFP steady-state levels, switching to active $P_{R}$ promoter (Fig. 2A, state 3). To check the stability of the activated $P_{R}$ promoter, we first expressed the proteins in the monostable region at $40^{\circ} \mathrm{C}$ for $1 \mathrm{~h}$, followed by a drop to lower temperatures. $P_{R}$ activity was reliably maintained (Fig. 2B, lower panel and Fig. S7), independently of the compartment geometry and hence protein lifetime (Fig. S9 and Fig. S8). Control experiments with a GRN lacking the cro gene (a monostable GRN) resulted in a similar initial response to temperature variations, but with overall lower GFP levels due to the lack of commitment to the $\mathrm{P}_{\mathrm{R}}$ promoter by Cro, as was already observed in bacteria (Fig. S9) (18), suggesting that Cro was required to obtain decisive switching (Fig. 2B, lower panel). Overall we observed a deterministic response to the temperature input, most likely due to low noise levels, with precise but slow dynamics on a characteristic time-scale of $\sim 1 \mathrm{~h}$ (Fig. S10), consistent with the bistable feature of the GRN as an ideal memory device. Solution experiments reconstituted a similar temperature response of the different GRNs, but could not identify a decisive switching temperature since the closed system accumulated proteins without turnover, and hence did not reach steady-state dynamics (Fig. S11).

In the low-copy regime, with $\mathrm{Cl}^{\mathrm{ts}}$-mVenus reporting on the $\mathrm{P}_{\mathrm{RM}}$ activity, the overall production rates of an ensemble of compartments (Fig. S12, Fig. 2C, right column and Table S2) increased (Fig. 2A, state 2) and decreased again at higher temperature as anticipated (Fig. 2C and Fig. 2A, state 3). We observed variability between compartments only in the $35-39{ }^{\circ} \mathrm{C}$ range as computed from the standard deviation of time-averaged production rates (Fig. S13). In this range, production rates on the time scale of many minutes ( $\sim \mathrm{min}$ ) waned and grew again (Fig. $2 \mathrm{C}$, Fig. S10, Fig. S12). In the absence of Cro, we expected to observe a $P_{\text {RM }}$ activity without inhibition by Cro, that is no display of spontaneous transitions between the two promoters. Supportively, the temperature response for the monostable GRN was similar to the bistable GRN (Fig. 3A,B and 2C, respectively and Fig. S12), but occurred across a narrower temperature range with higher median production rate at $37^{\circ} \mathrm{C}$ (Fig. 2C, Fig. $3 \mathrm{~A}$, and Table S2), and reduced variability at $37^{\circ} \mathrm{C}$ and $39^{\circ} \mathrm{C}$ 
(Fig. S13). A control experiment of a monostable GRN with the wild-type $\mathrm{cl}$ gene displayed almost no temperature response (Fig. S14 and Table S2). To exclude the possibility that the high concentration of the TXTL machinery in the cell-free extract (25) governed the GRN dynamics, we replaced the $P_{R M}$ by a consensus promoter sequence and added a ribosomal binding site (RBS) to the natural leaderless mRNA (Fig. S15) (26). We observed a strong increase in production rates in both cases. These control experiments supported $\mathrm{P}_{\mathrm{RM}}$ activity as the rate limiting step of the GRN, also validating $\mathrm{Cl}$ as the main regulator of $\mathrm{P}_{\mathrm{RM}}$. We also found no dependence of protein production rates on the slightly varying number of DNA molecules in the low-density compartments (Fig. S2). The data therefore suggests that in the bistable GRN either one of the two promoters was randomly activated and could spontaneously transition to the other promoter, but only at the $35-39{ }^{\circ} \mathrm{C}$ temperature range (20). We also note that the variability occurred in a broad temperature range close to the switching point of the high-density regime.

Whereas computational speed seemed to be traded for precision at an energetic cost of $\sim 10^{6}$ proteins in the high-density regime, fuzzy computation led to increase in speed with only a handful of proteins in the low-density regime (Fig. S10). Still, we wondered how decisions could be realized in the low-density regime considering the low DNA and protein concentration. We estimated the total number of proteins produced in 50 min to be $\sim 1000$, which amounted to an upper-limit concentration of $\sim 50 \mathrm{pM}$. With DNA at $10 \mathrm{pM}$, the concentrations were below the measured $\mathrm{Cl}$ binding affinities of $\sim 3 \mathrm{nM}(27)$ and $\sim 50 \mathrm{nM}(12)$, in vitro and in vivo, respectively, suggesting that equilibrium binding considerations could not account for the binding of nascent $\mathrm{Cl}^{\text {ts }}$ to its DNA binding site. Considering the typical binding rate of $\mathrm{Cl}$ to its operator sites of $\mathrm{k}_{\mathrm{on}} \approx 310^{7} \mathrm{M}^{-1} \mathrm{~s}^{-1}(28)$, it would take a single protein much more than 20 min to bind and modulate production rates, slower than the observed time to reach steady-states. These considerations could be reconciled by a scenario in which regulatory proteins are localized to the DNA during production and kept close to their operator sites with an increase in local effective concentration (Fig. $3 \mathrm{C}$ ). Since our system did not allow us to observe promoter activity, mRNA production, and protein synthesis at the same time and space, we corroborated this notion by stochastic simulations and found that they reproduced the experimental results only 
at values 100 -fold higher than previously measured $\mathrm{Cl} \mathrm{k}_{\mathrm{on}}$ rates in equilibrium (compare Fig. 3A to Fig. 3D and Table S3). Likewise, $\mathrm{Cl}^{\mathrm{ts}}$ repressed the production of GFP in solution experiments more efficiently when produced on the same plasmid than on a separate plasmid (Fig. S16). This notion of spatial coupling between protein synthesis and DNA-binding as an enhancement mechanism for site location was also discussed in a bacterial context (29) but was not demonstrated in vitro so far.

Next, we sought to find the source for the fuzzy decision-making in the low-density regime. The Fano factor was computed as a measure of fluctuations in $\mathrm{Cl}^{\text {ts }}-\mathrm{mVenus}$ production rates in single compartments after $20 \mathrm{~min}$ (the variance/mean $=1$ for a Poissonian process) and plotted in Fig. 4A. The Fano factor was close to 1 and constant with temperature for the wild-type monostable GRN. For both mono- and bistable GRNs, the Fano factor was greater than 1 around $37^{\circ} \mathrm{C}$, and correlated with the time-averaged production rates (Fig. 4A, upper row, Fig. S17). In addition, the Fano factor of the bistable GRN had a larger spread between $35-39{ }^{\circ} \mathrm{C}$ in the range of large variability. The data indicated that the regulation of the $\mathrm{P}_{\mathrm{RM}}$ activity by $\mathrm{Cl}^{\mathrm{ts}}$ mVenus strongly fluctuated only around $37^{\circ} \mathrm{C}$. Here, the fast inactivation and therefore lower occupancy of $\mathrm{Cl}^{\text {ts }}$-mVenus at the $\mathrm{P}_{\mathrm{R}}$ promoter seemed to reduce the stability of the auto-activation of $\mathrm{P}_{\mathrm{RM}}$ activity by $\mathrm{Cl}^{\mathrm{ts}}$-mVenus (Fig. 4B and Fig. S18); hence a lower occupancy at the $\mathrm{P}_{\mathrm{R}}$ promoter allows the leaky production of Cro to induce the observed variability and spontaneous transitions between promoters in the bistable GRN, resulting in fuzzy decision-making.

To extract typical correlation time scales in the fluctuating production rates of $\mathrm{Cl}^{\text {ts }}$ mVenus after $20 \mathrm{~min}$, we computed the autocorrelation functions (ACF) in each compartment. The ensemble-averaged ACFs decayed within a few minutes as expected for the random protein synthesis from 20 DNA molecules (Fig. S17) (23). However, the ACF amplitude at a delay of $\tau=30$ seconds had a higher amplitude for the bistable GRN than the wild-type and monostable GRN at $39^{\circ} \mathrm{C}$ and below (Fig. $4 \mathrm{~A}$, lower row and Fig. S17). This may indicate more correlated fluctuations of $\mathrm{Cl}^{\mathrm{ts}}$ mVenus production rates for the bistable GRN as was observed for a close bifurcation point in other complex dynamic systems, and can be explained by a 
reduced recovery rate from perturbations (30). Here, molecular perturbations may stem from the leaky production of Cro from $P_{R}$ within the region of bistability (Fig. 4B). To get better statistics on the long-term fluctuations that could last for many minutes in the bistable GRN (Fig. 2C), we extended the duration of the experiment to $130 \mathrm{~min}$ at $37^{\circ} \mathrm{C}$ (Fig. 4C). We observed highly fluctuating production rates with repeating signatures of protein bursts, each lasting for several minutes (Fig. 4D and Fig. S19). A strongly damped periodic signal could be observed in individual ACFs with a period of $\sim 10 \mathrm{~min}$, but averaged out when ensemble-averaged (Fig. S18).

We established a minimal decision-making GRN, with and without variability and spontaneous transitions, in a synthetic cell devoid of any extrinsic noise. Dynamics could be monitored at the low and high copy number limits of genetic computation, demonstrating a clear tradeoff between slow and precise versus fast and fuzzy. We discovered evidence for enhanced rates by co-expressional localization (Fig. 3) (29), a nonequilibrium mechanism that seems to be essential for realizing single-molecule regulation in our minimal cell models. 


\section{A} Noise No. of computing particles

1.
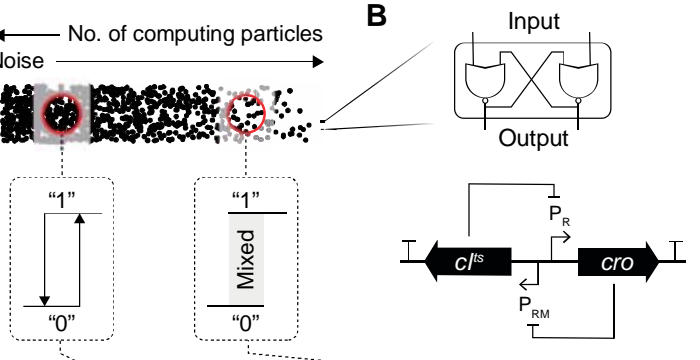

C
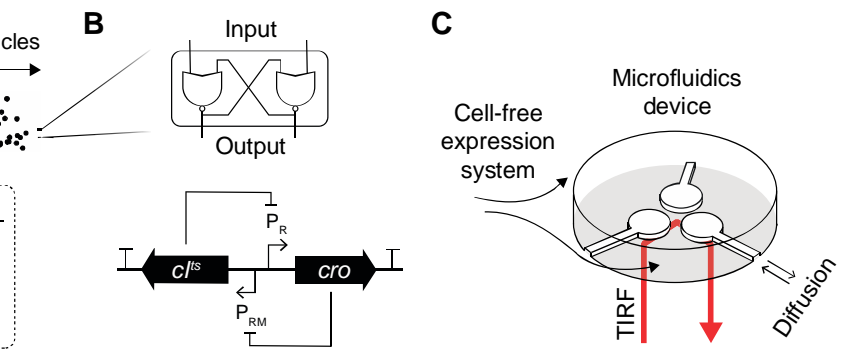

D Noiseless regime, Genes $\sim 10^{6} \mathbf{x}$
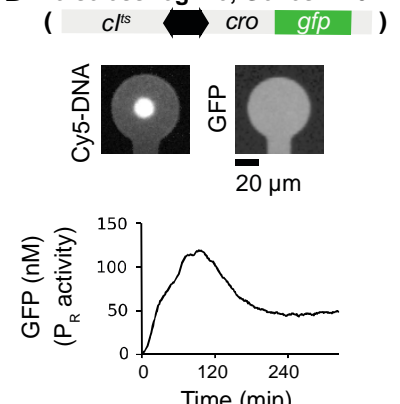

Time $(\min )$

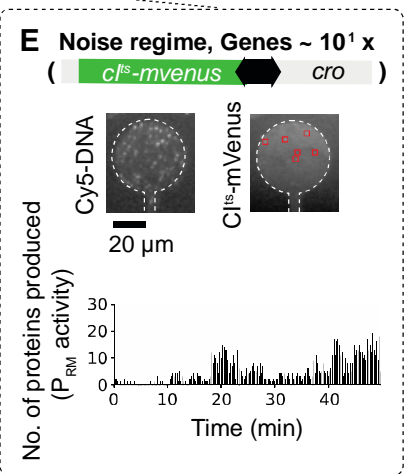

Fig. 1. Biological computation in a synthetic cell model at high and low gene numbers. (A) Number of computing particles influence decision-making by an effective noise level: a bistable GRN responds to an input with a binary output in the noiseless regime at large numbers, or by a fuzzy-logic computation with mixed outputs in the noise regime at small numbers. (B) Scheme of the minimal bistable GRN based on $\mathrm{Cl}$ and Cro transcription factors repressing each other's promoter with analogy to an electronic latch circuit. (C) Scheme of a microfluidic device with three compartments as synthetic cell models. (D) Cy5-DNA assembled in a compartment at high density gives rise to GFP expression reporting on the $P_{R}$ activity. (E) Cy5-DNA at single molecule resolution inside the compartment. Single $\mathrm{Cl}^{\text {ts }}$-mVenus were integrated over the compartment to give number of proteins produced in 15 seconds, reporting on the overall $\mathrm{P}_{\mathrm{RM}}$ activity. 
A

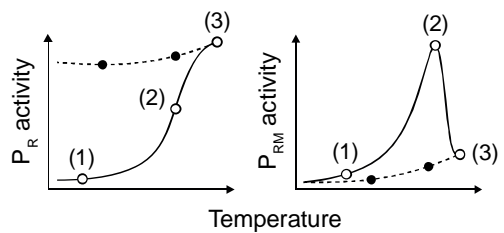

(1)

(2)

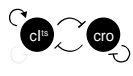

(3)

B Noiseless regime, Genes $\sim 10^{6} \mathrm{x}$

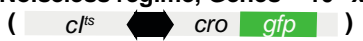

C Noise regime, Genes $\sim 10^{1} \mathrm{x}$
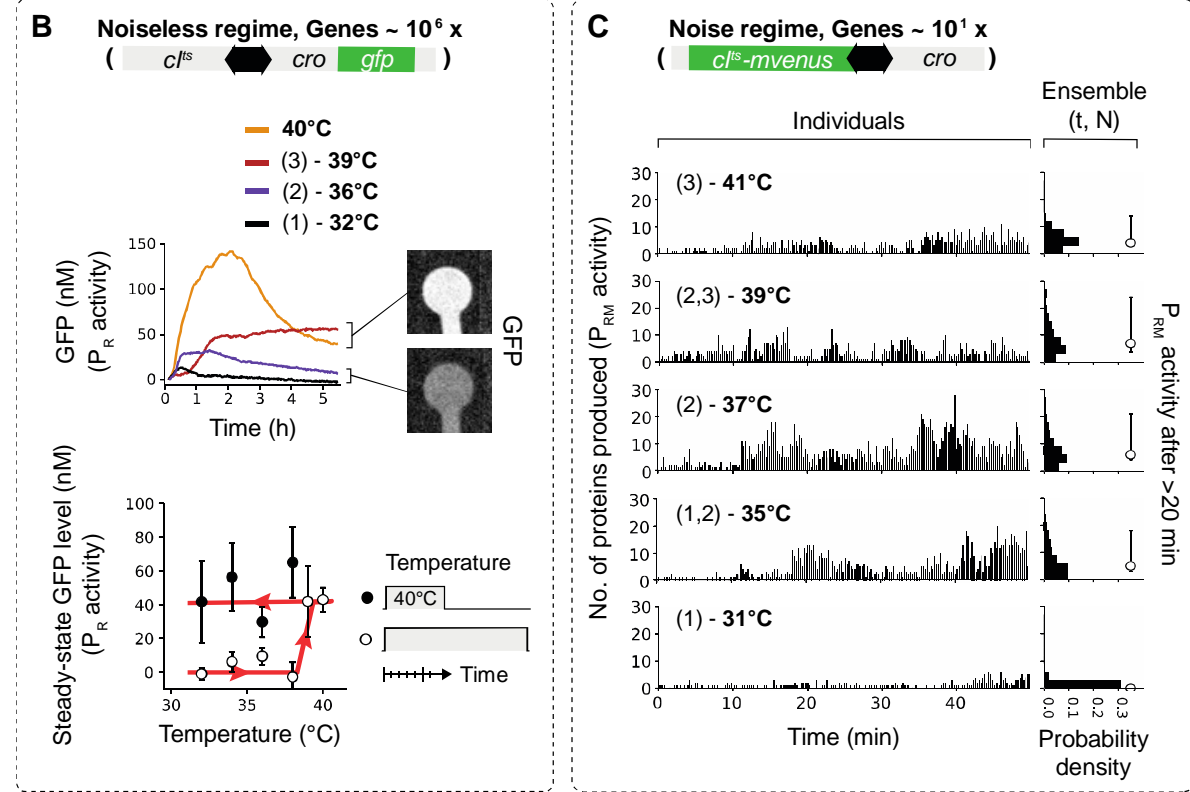

Fig. 2. Bistable GRN and decision-making at high and low gene densities. (A) Illustration for the expected output of $\mathrm{P}_{\mathrm{R}}$ and $\mathrm{P}_{\mathrm{RM}}$ activity as a function of temperature, based on the known molecular feedbacks of the GRN. (B) Dynamics of GFP expression reporting on the $P_{R}$ activity at high DNA density (upper panel). Solid lines depict average dynamics, $\mathrm{N}=24$. (1), (2), (3) relate to molecular states of the bistable GRN at each temperature, as in (A). Steady-state GFP levels averaged over individual compartments (lower panel). Temperature was either kept constant throughout the measurement (open circle) or after a $40^{\circ} \mathrm{C}$ initial incubation step (closed circles). Error bars show standard deviation (SD) of compartments. (C) Number of $\mathrm{Cl}^{\mathrm{ts}}$-mVenus produced in 15 seconds to give production rates (left column) and ensemble distribution over time $t$ and compartments $\mathrm{N}$ after $>20$ min (right column) in the low-copy regime. Circle with error bars give the median and 32th to 68th percentile of the ensemble production rates. 
A
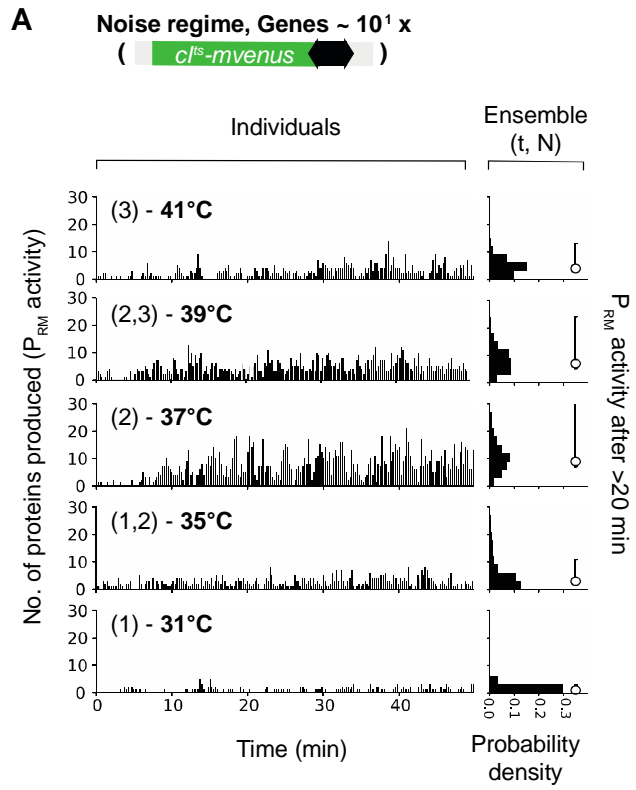

B
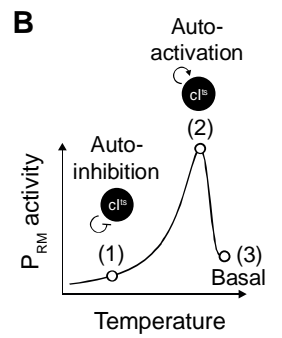

D Model ensemble ( $t, N)-P_{\text {RM }}$ activity

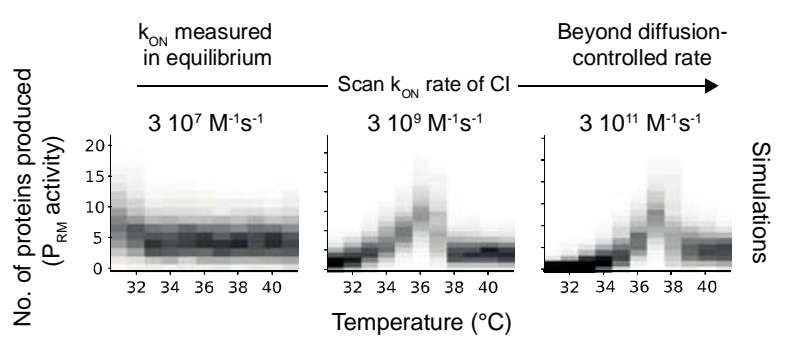

Fig. 3. Monostable GRN and co-expressional localization at the low-density limit. (A) Production rate of $\mathrm{Cl}^{\text {ts }}$-mVenus proteins in 15 seconds inside individual compartments (left column) and ensemble production rates (right column) as in Fig. $2 \mathrm{C}$. Circle with error bars give the median and the 32th to 68th percentile of the ensemble production rates. (B) Illustration of the expected $P_{R M}$ activity with temperature in the monostable GRN and the corresponding states. (C) A protein repressor synthesized in proximity to its DNA binding site, reach higher effective concentrations by coexpression localization (left scheme) than in an equilibrium scenario due to diffusion away from the DNA (right scheme). (D) Ensemble production rates obtained from stochastic simulations with a scan in the $\mathrm{Cl}^{\text {ts }}$ binding rate to the DNA binding sites. 
A Fluctuation properties
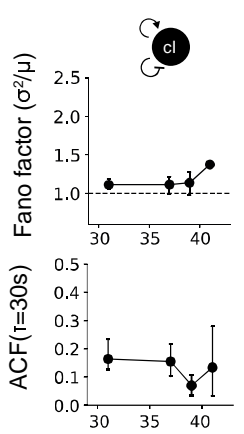

C

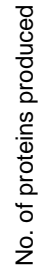
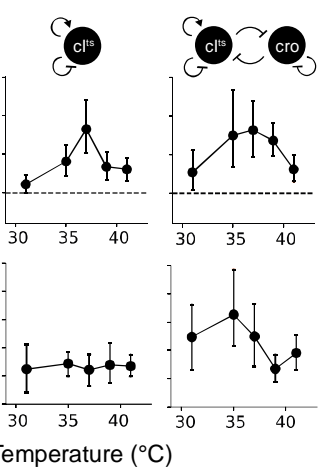

Temperature $\left({ }^{\circ} \mathrm{C}\right)$

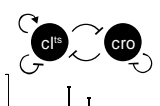

B
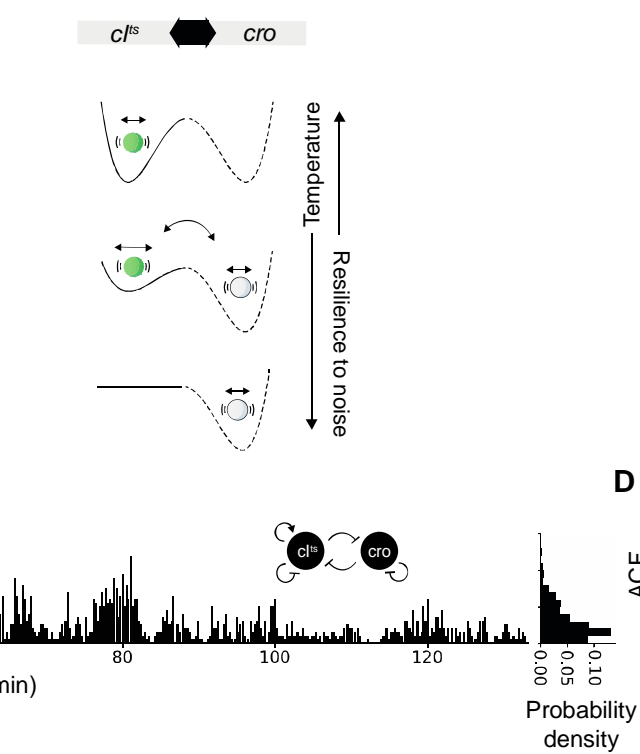

D

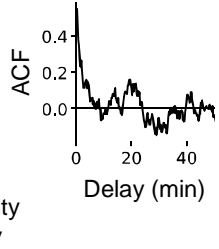

Fig. 4. Fluctuations and spontaneous transitions at the low-density limit. (A) Fano factor (variance over mean) with Poissonian process (dashed line) for the GRNs at various temperatures (upper row). Amplitude of temporal auto-correlation function (ACF) of fluctuating production rates (Fig 2C, 3A, Fig S16) in individual compartments at $\tau=30 \mathrm{~s}$. Error bars were bootstrapped and show SD of compartments. (B) Scheme of noise, active promoter transitions and memory in the bistable GRN. Short-term fluctuations in gene expression (circle within a well) originate from local noise. Long-term fluctuations can originate from spontaneous promoter transitions with the deactivation of $\mathrm{Cl}^{\text {ts }}$. (C) Number of proteins produced in 15 seconds for long-term experiment of the bistable GRN at $37^{\circ} \mathrm{C}$. (D) The ACF of the trace in (C). 


\section{References and Notes}

1. Reed, A. V. Speed-accuracy trade-off in recognition memory. Science 181, 574-576 (1973).

2. Hopfield, J. J. Kinetic Proofreading: A New Mechanism for Reducing Errors in Biosynthetic Processes Requiring High Specificity. Proc. Natl. Acad. Sci. 71, 4135-4139 (1974).

3. Savir, Y. \& Tlusty, T. The Ribosome as an Optimal Decoder: A Lesson in Molecular Recognition. Cell 153, 471-479 (2013).

4. Lan, G., Sartori, P., Neumann, S., Sourjik, V. \& Tu, Y. The energy-speedaccuracy trade-off in sensory adaptation. Nat. Phys. 8, 422-428 (2012).

5. Lestas, I., Vinnicombe, G. \& Paulsson, J. Fundamental limits on the suppression of molecular fluctuations. Nature 467, 174-178 (2010).

6. Taniguchi, Y. et al. Quantifying E. coli proteome and transcriptome with singlemolecule sensitivity in single cells. Science 329, 533-538 (2010).

7. Bialek, W. Stability and noise in biochemical switches. Adv. Neural Inf. Process. Syst. 13, 103-109 (2001).

8. Warren, P. B. \& Ten Wolde, P. R. Enhancement of the stability of genetic switches by overlapping upstream regulatory domains. Phys. Rev. Lett. 92, 14 (2004).

9. Lord, N. D. et al. Stochastic antagonism between two proteins governs a bacterial cell fate switch. Science 366, 116-120 (2019).

10. Sepúlveda, L. A., Xu, H., Zhang, J., Wang, M. \& Golding, I. Measurement of gene regulation in individual cells reveals rapid switching between promoter states. Science 351, 1218-1222 (2016).

11. St-Pierre, F. \& Endy, D. Determination of cell fate selection during phage lambda infection. Proc. Natl. Acad. Sci. U. S. A. 105, 20705-20710 (2008).

12. Rosenfeld, N., Young, J. W., Alon, U., Swain, P. S. \& Elowitz, M. B. Gene regulation at the single-cell level. Science 307, 1962-1965 (2005).

13. Karzbrun, E., Tayar, A. M., Noireaux, V. \& Bar-Ziv, R. H. Programmable onchip DNA compartments as artificial cells. Science 345, 829-832 (2014).

14. Zeng, L. et al. Decision Making at a Subcellular Level Determines the Outcome of Bacteriophage Infection. Cell 141, 682-691 (2010).

15. Arkin, A., Ross, J. \& McAdams, H. H. Stochastic kinetic analysis of developmental pathway bifurcation in phage $\lambda$-infected Escherichia coli cells. 
Genetics 149, 1633-1648 (1998).

16. Ptashne, M. A genetic switch 3rd edition. CSHL Press (2004). doi:10.1038/nrc1424

17. Norman, T. M., Lord, N. D., Paulsson, J. \& Losick, R. Stochastic Switching of Cell Fate in Microbes. Annu. Rev. Microbiol. 69, 381-403 (2015).

18. Schubert, R. A., Dodd, I. B., Egan, J. B. \& Shearwin, K. E. Cro's role in the ClCro bistable switch is critical for $\lambda$ 's transition from lysogeny to lytic development. Genes Dev. 21, 2461-2472 (2007).

19. Isaacs, F. J., Hasty, J., Cantor, C. R. \& Collins, J. J. Prediction and measurement of an autoregulatory genetic module. Proc. Natl. Acad. Sci. U. S. A. 100, 7714-7719 (2003).

20. Fang, X. et al. Cell fate potentials and switching kinetics uncovered in a classic bistable genetic switch. Nat. Commun. 9, 2787 (2018).

21. Buxboim, A., Daube, S. S. \& Ziv, R. B. Synthetic gene brushes: a structurefunction relationship. Mol. Syst. Biol. 4, 181 (2008).

22. Balleza, E., Kim, J. M. \& Cluzel, P. Systematic characterization of maturation time of fluorescent proteins in living cells. Nat. Methods 15, 47-51 (2018).

23. Yu, J., Xiao, J., Ren, X., Lao, K. \& Xie, X. S. Probing gene expression in live cells, one protein molecule at a time. Science 311, 1600-1603 (2006).

24. Hensel, Z. et al. Stochastic expression dynamics of a transcription factor revealed by single-molecule noise analysis. Nat. Struct. Mol. Biol. 19, 797-802 (2012).

25. Garamella, J., Marshall, R., Rustad, M. \& Noireaux, V. The All E. coli TX-TL Toolbox 2.0: A Platform for Cell-Free Synthetic Biology. ACS Synth. Biol. 5, 344-355 (2016).

26. Moll, I., Grill, S., Gualerzi, C. O. \& Bläsi, U. Leaderless mRNAs in bacteria: Surprises in ribosomal recruitment and translational control. Mol. Microbiol. 43, 239-246 (2002).

27. Johnson, A. D., Meyer, B. J. \& Ptashne, M. Interactions between DNA-bound repressors govern regulation by the $\lambda$ phage repressor. Proc. Natl. Acad. Sci. U. S. A. 76, 5061-5065 (1979).

28. Nelson, H. C. M. \& Sauer, R. T. Lambda repressor mutations that increase the affinity and specificity of operator binding. Cell 42, 549-558 (1985).

29. Kolesov, G., Wunderlich, Z., Laikova, O. N., Gelfand, M. S. \& Mirny, L. A. How 
gene order is influenced by the biophysics of transcription regulation. Proc.

Natl. Acad. Sci. U. S. A. 104, 13948-13953 (2007).

30. Scheffer, M. et al. Early-warning signals for critical transitions. Nature 461, 5359 (2009).

31. Marshall, R. \& Noireaux, V. Quantitative modeling of transcription and translation of an all-E. coli cell-free system. Sci. Rep. 9, (2019).

32. Sun, Z. Z., Yeung, E., Hayes, C. A., Noireaux, V. \& Murray, R. M. Linear DNA for rapid prototyping of synthetic biological circuits in an escherichia coli based TX-TL cell-free system. ACS Synth. Biol. 3, 387-397 (2014).

33. Mukherjee, A., Walker, J., Weyant, K. B. \& Schroeder, C. M. Characterization of flavin-based fluorescent proteins: an emerging class of fluorescent reporters. PLoS One 8, e64753 (2013).

34. Buxboim, A. et al. A single-step photolithographic interface for cell-free gene expression and active biochips. Small 3, 500-510 (2007).

35. Nicovich, P. R., Walsh, J., Böcking, T. \& Gaus, K. NicoLase - An open-source diode laser combiner, fiber launch, and sequencing controller for fluorescence microscopy. PLoS One 12, 1-15 (2017).

36. Schnitzbauer, J., Strauss, M. T., Schlichthaerle, T., Schueder, F. \& Jungmann, R. Super-resolution microscopy with DNA-PAINT. Nat. Protoc. 12, 1198-1228 (2017).

37. Hua, B. et al. An improved surface passivation method for single-molecule studies. Nat. Methods 11, 1233-1236 (2014).

38. Karzbrun, E., Shin, J., Bar-Ziv, R. H. \& Noireaux, V. Coarse-grained dynamics of protein synthesis in a cell-free system. Phys. Rev. Lett. 106, 48104 (2011). 
Acknowledgments: We thank M. Levy and O. Vonshak for fruitful discussions and help with experiments; L Tunik for help in the fabrication facilities; N. Stern, M. Schwarz-Schilling, and T. Tlusty for helpful comments on the manuscript; and J. Götz for the brilliant support on all levels throughout the project. We thank D. Garenne for his participation in the preparation of the TXTL system used in this work. pGEX iLOV was a gift from John Christie (Addgene plasmid \#26587; http://n2t.net/addgene:26587; RRID:Addgene_26587). Funding: We acknowledge funding from the Israel Science Foundation (RBZ and SSD, grant no. 1870/15), the United States - Israel Binational Science Foundation (RBZ and VN grant no. 2014400), and the Minerva Foundation (RBZ and SSD, grant no. 712274). We also thank EMBO for the financial support of F.G. through a long-term fellowship (ALTF 598-2017). Author contributions: F.G. designed the system, performed experiments and analyzed the data. All authors discussed data and wrote the manuscript. Competing interests: The Noireaux laboratory receives research funds from Arbor Biosciences, a distributor of the myTXTL cell-free protein synthesis kit.

\section{Supplementary Materials}

Materials and Methods

Figures S1-S19

Tables S1-S3 